\title{
STRATEGY TO MASK THE BITTER TASTE OF MOMORDICA CHARANTIA EXTRACT USING ALGINATE-GELATIN BEADS
}

\author{
SUTRIYO*, RADITYA ISWANDANA, FAUZANA FAUZI
}

Laboratory of Pharmaceutics and Pharmaceutical Technology Development, Faculty of Pharmacy, Universitas Indonesia, Depok, 16424, Indonesia. Email: sutriyo@farmasi.ui.ac.id

Received: 26 April 2018, Revised: 28 September 2018, Accepted: 09 November 2018

\section{ABSTRACT}

Objective: This study is aimed to develop a strategy to mask the bitter taste of Momordica charantia Linn. extract using alginate-gelatin beads.

Methods: Beads of bitter melon fruit extract (M. charantia Linn) were prepared using an ionic gelation method in which cross-linking occurs between sodium alginate and calcium chloride that serves to mask the bitter taste. Beads were prepared using sodium alginate (1.5\% w/v) with various concentrations of bitter melon fruit extract $(1: 2,1: 1$, and $1: 0.5)$, gelatin $(2 \% \mathrm{w} / \mathrm{v})$, and $\mathrm{CaCl} 23 \%$. The obtained beads were then characterized both physically and functionally, and their morphology, process efficiency, particle distribution, swelling index, and water content were recorded.

Results: Formula 1 beads, with a 1:2 ratio of extract: alginate, displayed a near-round shape with a diameter of 600-1 200 $\mu$ m, a swelling index of $113.21 \%$, and a moisture content of $15.34 \%$. In addition, these beads were able to significantly cover the bitter taste ( $\mathrm{p}<0.05$ ).

Conclusion: Formula 1 beads with a 1:2 ratio of extract: alginate can effectively mask the bitter taste of $M$. charantia Linn.

Keywords: Alginate, Beads, Bitter melon extract (Momordica charantia Linn.), Bitter taste masking, Gelatin.

(C) 2018 The Authors. Published by Innovare Academic Sciences Pvt Ltd. This is an open access article under the CC BY license (http://creativecommons. org/licenses/by/4. 0/) DOI: http://dx.doi.org/10.22159/ijap.2018.v10s1.84

\section{INTRODUCTION}

One of the natural ingredients of Indonesia that is used for various treatments is bitter melon (Momordica charantia Linn). In the past two decades, many studies have confirmed the pharmacological effects of bitter melon, including its antidiabetic, anticancer, antiviral, antioxidant properties, as well as its action as an $\alpha$-amylase and ACE inhibitor [1-4]. The pharmacological effects are believed to be due to the active compounds present in the extract, including polysaccharide sulfate derivatives, guluronic acid, galactic acid, hexosamine and neutral (D-galactose) or uronic acid (D-glucoronic or L-iduronic acid) polysaccharides, and other secondary compounds such as alkaloids, flavonoids, and phenols [5].

Despite its numerous health benefits, one disadvantage to bitter melon extract is that it is extremely bitter tasting. The bitter taste is produced by triterpene glycosidase compounds such as $\mathrm{K}$ and $\mathrm{L}$ momordicosida, momordicin, and cucurbitacin [6-8]. Therefore, in this study, we sought to improve patient compliance by coating the active components with a compatible compound in a process known as microencapsulation. The method used was ion gelation using sodium alginate and gelatin polymer.

Alginate is a natural polymer found in chocolate algae extract (Phaeophyceae) and is biodegradable, biocompatible, non-toxic, and hydrophilic, thus suitable for coating a water-soluble active compound. The alginate polymer has two chains: $1.4 \beta$-D-manuronic acid (M) and 1,4 $\alpha$-L-guluronic acid (G), which can form bonds with each other (homopolymer) or both (heteropolymer) [9]. Monovalent metal ions will form water-soluble alginate salts, while divalent and multivalent metals, with the exception of $\mathrm{Mg}^{2+}$, will form a water-insoluble gel. Alginate with a high guluronic acid (G) content will cross-link with divalent ions such as $\mathrm{Ca}^{2+}[10]$. Cross-linking with calcium ions serves to decrease the expansion and permeability of alginates, which leads to a decrease in the release of extracts when consumed so as to minimize the bitter taste. This process, involving the cross-linking of alginate with calcium ions, combined with gelatin, should retain gel strength and produce good beads [11].

\section{MATERIALS AND METHODS}

Materials

Bitter melon extract (M. charantia Linn) (Indonesia), sodium alginate (Asian Chemical, Indonesia), calcium chloride (Asian Chemical, Indonesia), gelatin type B (Brataco, Indonesia), and aqua demineralized (Brataco, Indonesia) were used.

\section{Optimization of gelatin concentrations}

The sodium alginate solution was prepared by dissolving $1.5 \mathrm{~g}$ into $50 \mathrm{~mL}$ demineralized water and stirred while heating at $100^{\circ} \mathrm{C}$. Gelatin at different concentrations $(1 \mathrm{~g}, 1.5 \mathrm{~g}$, and $2 \mathrm{~g}$ ) was dissolved into $50 \mathrm{~mL}$ demineralized water and stirred while heating at $40^{\circ} \mathrm{C}$. Then, the sodium alginate solution was mixed into the gelatin until homogenous. The polymer mixture was then fed into a $26-\mathrm{G}$ syringe needle and dripped into a $3 \%$ calcium chloride solution while stirring with a magnetic stirrer at $200 \mathrm{rpm}$ and allowed to stand for $15 \mathrm{~min}$. The beads were then filtered and washed with demineralized water and dried (Table 1).

\section{Bitter melon fruit extract beads}

The bitter melon extract was dissolved into demineralized water and then mixed into a polymer solution. The sodium alginate-gelatin extract mixture was then dropped using a syringe needle into a $3 \%$ calcium chloride solution while stirring with a magnetic stirrer at $200 \mathrm{rpm}$ and allowed to stand for $15 \mathrm{~min}$. The beads were then filtered and washed with demineralized water and then dried.

\section{Morphology}

The shape and surface morphology of the beads was observed using a scanning electron microscope (SEM). Dry beads were placed on the sample holder and analyzed under vacuum.

\section{Particle size distribution}

The particle size distribution of the beads was evaluated using calipers. A total number of 300 beads were measured for their diameter and the calculated percentage of the size for each formula. 
Table 1: Bead formulation with bitter melon extract

\begin{tabular}{ll}
\hline Code & Extract and sodium alginate ratio \\
\hline F1 & $1: 2.0$ \\
F2 & $1: 1.0$ \\
F3 & $1: 0.5$ \\
\hline
\end{tabular}

\section{Moisture content}

The moisture content was analyzed using a moisture content analyzer, which was preheated for $3 \mathrm{~min}$, and then the temperature was set to $105^{\circ} \mathrm{C} .1 \mathrm{~g}$ of beads is then placed on top of the aluminum in the tool, and the value of the water content is read after reaching a constant value.

\section{Process efficiency}

The process efficiency was determined by comparing the total weight of the beads obtained with the total material used for the manufacture of the beads. The value was then measured using the equation as follows:

$$
\frac{\text { Weight of the dried beads (gram) }}{\text { Total weight of material used (gram) }} \times 100 \%
$$

\section{Swelling index}

As many as $100 \mathrm{mg}\left(\mathrm{W}_{0}\right)$, the beads were soaked in a beaker glass containing $10 \mathrm{~mL}$ demineralized water. After $5 \mathrm{~min}$, the beads were removed from the medium and weighed $\left(\mathrm{W}_{1}\right)$. The same procedure was performed at 10, 15, 30, 45, and 60 min intervals. The swelling index of the beads was calculated by dividing $\left(\mathrm{W}_{1}-\mathrm{W}_{2}\right)$ with $\mathrm{W}_{0}$ then times $100 \%$.

\section{Fourier-transform infrared (FT-IR)}

A total of $2 \mathrm{mg}$ of dried $\mathrm{KBr}$ and $98 \mathrm{mg}$ of sample were mixed homogeneously and analyzed by FT-IR at wave numbers of $400-4000 \mathrm{~cm}^{-1}$. The IR spectra obtained from the beads was compared with each IR spectrum of alginate and gelatin.

\section{Bitter taste evaluation}

Three formulas of beads (Formulas 1-3) and a solution of bitter melon extract (as the control standard) were tested by 30 male and female respondents, aged 20-30 years. The participants were screened before the study and those with mouth disorders, such as canker sores, bleeding gums, or toothaches, or who were smokers, were excluded to minimize any influences on the sense of taste. The sample was placed into the mouth and allowed to stand for $10 \mathrm{~s}$. The sample was then removed, and the mouth was washed with water. Respondents then gave a bitter taste value of the sample according to the following scale: Value 0 for non-bitter, value 1 for bit bitter, value 2 for bitter, and value 3 for very bitter. After a 10-min break, the same procedure was performed again for the next sample. Assessment results were analyzed using the IBM SPSS statistics program.

\section{RESULTS AND DISCUSSION}

\section{Optimization of gelatin concentrations}

The combination with gelatin $2 \%$ produced a more rounded shape of bead compared with the alginate beads because the gelatin can retain the shape of the beads (Fig. 1). In the presence of ionic bonds, the hydrogen bonds between the guluronic acid and amino acids in the gelatin increased the density of the beads. The beads that only used alginate had a looser structure after passing through the drying process [12].

\section{Morphology}

Test results of the bead formulas using scanning electron microscopy showed almost spherical, filamentous, and uneven surfaces (Fig. 2). This was caused by the extract of the bitter melon, which is hygroscopic, and thus, after going through the drying process, it can draw water back causing the non-spherical shape.

\section{Particle size distribution}

The diameter of the particle size was in the range of 620-1180 $\mu \mathrm{m}$, and the SEM results are shown in Fig. 2. The particle size of the beads varied
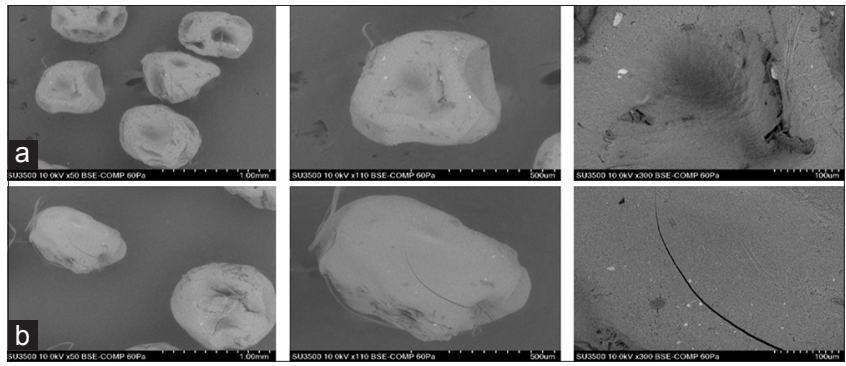

Fig. 1: Scanning electron microscope results at $\times 50, \times 100$, and $\times 300$ magnification, (a) alginate, (b) alginate-gelatin
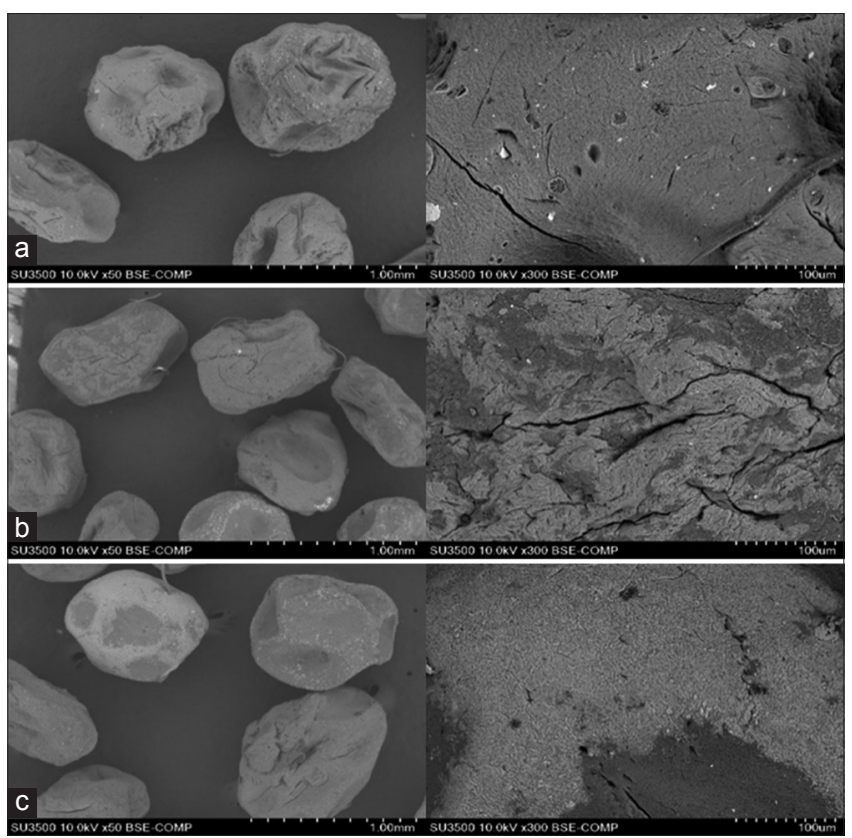

Fig. 2: Scanning electron microscope results of bitter melon extract beads. (a) Formula 1; (b) Formula 2; (c) Formula 3

because the process of making beads was done manually by dripping the coating mixer using a syringe needle. Therefore, the pressure and the angle were not the same, thus affecting the size of the beads coming out of the syringe needle [9].

\section{Moisture content}

The results of the moisture content analysis of the beads from the three formulas showed the amount of moisture content ranged from $14.56 \%$ to $18.86 \%$. The high moisture content was caused by the hygroscopic nature of the extract. The use of hydrophilic alginate polymers also made the beads which have a high moisture content [6].

\section{Process efficiency}

The process efficiency of the dry beads obtained from Formulas 1, 2, and 3 was $39.45 \%, 36.25 \%$, and $35.79 \%$, respectively. The more bitter melon extract used, the lower the efficiency of the manufacturing process. Bitter melon extract has a very high solubility in water so that when the process drips the polymer mixture and extract, part of the extract solution is dissolved in the calcium chloride solution during the cross-linking process [13].

\section{Swelling index}

According to Saarai et al., 2013, the swelling index of hydrogel compounds such as alginate and gelatin can be affected by the $\mathrm{pH}$ of the medium [12]. During this process, the acid and base groups of the polyelectrolyte compound will ionize as the $\mathrm{pH}$ medium changes. In Formula 1, the beads were inflated two-fold. In Formula 2, the beads were inflated two-fold but then lost their weight again, while in 


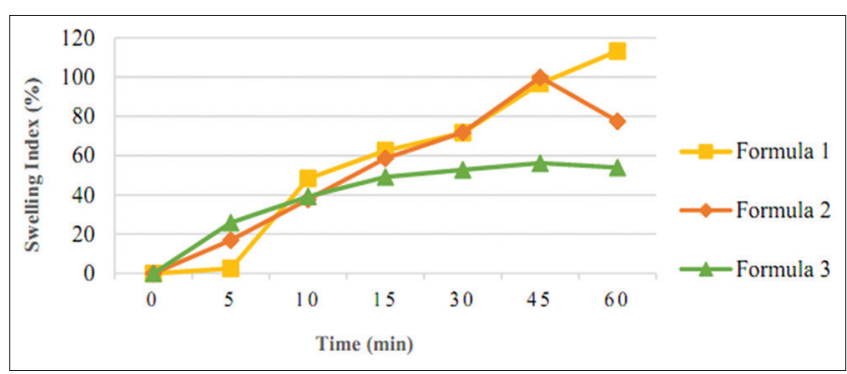

Fig. 3: Bead swelling index in demineralized water within $60 \mathrm{~min}$

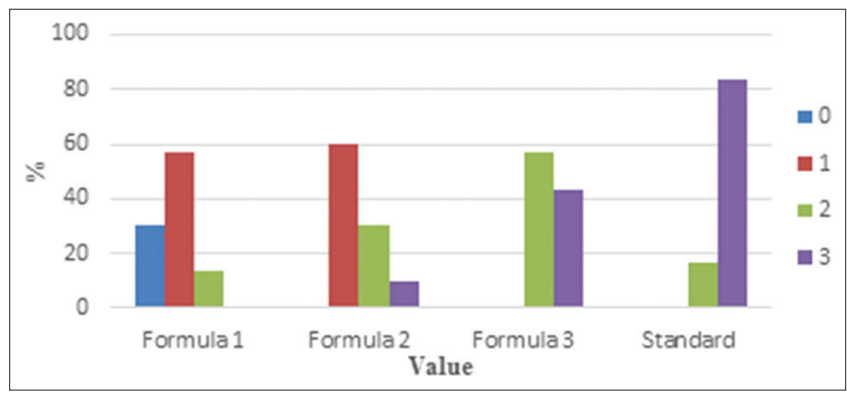

Fig. 4: Respondent percentage value of bitter melon samples and standard

Formula 3, the beads were only inflated 50\% (Fig. 3). This was because the amount of polymer present in the beads decreases with the increase of the bitter melon extracts so that the diffused water also decreases.

\section{FT-IR}

For the alginate FT-IR spectrum, there was a moderate intensity peak in the range of 1645 and $1452 \mathrm{~cm}^{-1}$ waves, indicating the presence of a carboxylic group ( $\mathrm{COO}^{-}$). For the spectrum of FT-IR gelatin, there was peak with moderate intensity in the range of wave number $1200-1650 \mathrm{~cm}^{-1}$, specifically, at $1452,1518,1553$, and $1640 \mathrm{~cm}^{-1}$, indicating the existence of an amide Group I and amide III (sharp NH and $\mathrm{CN}$ widen), and a $\mathrm{CO}$ group at $1050 \mathrm{~cm}^{-1}$ wave number, indicating the presence of an alkyl group. The wave number $3536 \mathrm{~cm}^{-1}$ indicates the presence of an $\mathrm{O}-\mathrm{H}$ group binding to an $\mathrm{N}-\mathrm{H}$ group [14].

From the FT-IR spectrum of empty beads, there was a peak of moderate intensity at $1438 \mathrm{~cm}^{-1}$ wave number, indicating the presence of alginate COO- groups attached to $\mathrm{Ca}^{2+}$ ions. This spectrum had a shift in the number of waves, becoming smaller. The $3525 \mathrm{~cm}^{-1}$ wave number indicated the presence of an $\mathrm{O}-\mathrm{H}$ group binding to an $\mathrm{N}-\mathrm{H}$ group on the decreased spectrum of gelatin. The wave numbers 1448, 1512, and $1552 \mathrm{~cm}^{-1}$ indicated the presence of amide groups that have decreased wave numbers [14].

The decrease of wave number was caused by the ionic bonds, as well as the hydrogen bonds formed between alginate and gelatin. The higher gelatine concentration that was used, the further shifting of the alginate spectral wave number, which results in a decreasing of the wave number [11,15-17].

\section{Bitter taste evaluation}

This test was performed to evaluate the respondent's assessment of the formula beads extract. The test results for Formula 1 indicated that most respondents gave a non-bitter value of $30 \%$ and bit bitter value of $56.67 \%$. For Formula 2, the values given by majority respondents were bitter (i.e., $60 \%$ ) and bitter as much as $30 \%$. For Formula 3, respondents gave a bitter value of $56.67 \%$ and a very bitter value of $43.33 \%$. As for the standard, most respondents gave a very bitter value of $83.33 \%$. These data are shown in Fig. 4.

Statistical analysis using the Wilcoxon test for the beads of the bitter melon extract and the standard showed that there was a significant difference in the bitter taste between the bitter melon extract of Formulas 1,2 , and 3 , with a significance value of $<0.05$. These results demonstrate that the extract of the bitter melon that was treated during the manufacture of the beads has a different taste than the standard bitter melon extract.

From the results of the statistical analysis and the percentage of the assessment, we can conclude that Formula 1 was the most optimal formula to mask the bitter taste of the bitter melon extract.

\section{CONCLUSION}

Formula 1 of the bitter melon beads, with a ratio of 1:2 (extract: alginate), had almost a round shape with a diameter of $600-1,200 \mu \mathrm{m}$, a swelling index of $113.21 \%$, and a moisture content of $15.34 \%$. In addition, this formula was able to significantly cover the bitter taste of the extract $(\mathrm{p}<0.05)$.

\section{CONFLICTS OF INTEREST}

All authors have none to declare.

\section{REFERENCES}

1. Xu X, Shan B, Liao CH, Xie JH, Wen PW, Shi JY. Anti-diabetic properties of Momordica charantia L. Polysaccharide in alloxaninduced diabetic mice. Int J Biol Macromol 2015;81:538-43.

2. Semiz A, Sen A. Antioxidant and chemoprotective properties of Momordica charantia L (bitter melon) fruit extract. Afr J Biotechnol 2007;6:273-7.

3. Puri M, Kaur I, Kanwar RK, Gupta RC, Chauhan A, Kanwar JR. Ribosome inactivating proteins (RIPs) from Momordica charantia for anti viral therapy. Curr Mol Med 2009;9:1080-94.

4. Tan H, Gan C. Polysaccharide with antioxidant, $\alpha$-amylase inhibitory and ACE inhibitory activities from Momordica charantia. Int J Biol Macromol 2016;85:487-96.

5. Zhang F, Lin L, Xie J. A mini-review of chemical and biological properties of polysaccharides from Momordica charantia. Int J Biol Macromol 2016;92:246-53.

6. Donya AB. The Effects of Various Processing Methods on the Momordicosides K and L Contents, and the Sensory Characteristics of Bitter Melon Vegetable (Momordica charantia L.). United States: University of Arkansas; 2007.

7. Gupta M, Sharma S, Gautam AK, Bhadauria R. Momordica charantia Linn. (Karela): Nature's silent healer. Int J Pharm Sci Rev Res 2011;11:32-7

8. Limtrakul P, Pitchakarn P, Suzuki S, Kuguacin J. A triterpenoid from Momordica charantia Linn: A comprehensive review of anticarcinogenic properties. Open Sci 2013;13:276-96.

9. Devi NKD, Chandana M, Sindhura A, Ratnavali G, Kavitha R. Comparative evaluation of alginate beads prepared by ionotropic gelation technique. Pharmacopore 2010;3:196-213.

10. Tønnesen HH, Karlsen J. Alginate in drug delivery systems. Drug Dev Ind Pharm 2002;28:621-30.

11. Dong Z, Wang Q, Du Y. Alginate/gelatin blend films and their properties for drug controlled release. J Memb Sci 2006;280: $37-44$.

12. Saarai A, Kasparkova V, Sedlacek T, Saha P. On the development and characterisation of crosslinked sodium alginate/gelatine hydrogels. J Mech Behav Biomed Mater 2013;18:152-66.

13. Kumar SR, Ashish J, Satish N. Momordica charantia Linn.: A mini review. Int J Manag Bus Res 2011;2:579-87.

14. Harmita. Textbook of Physicochemical Analysis. Depok: Department of Pharmacy, Faculty of Mathematics and Natural Sciences Universitas Indonesia; 2006

15. Namasivayam SK, Robin AT. Preparation, optimization and characterization of biocompatible nanoalbumin-ofloxacin (BSANP-OF) conjugate and evaluation of control release, anti bacterial activity against clinical isolate of Pseudomonas aeruginosa. Asian J Pharm Clin Res 2013;6:235-39.

16. Nagavarma BV, Hemant KS, Ayaz A, Vasudha LS, Shivakumar HG. Different techniques for preparation of polymeric nanoparticles - A review. Asian J Pharm Clin Res 2012;5:16-23.

17. Farshid A, Lakshmi CS. Formulation of nanoparticles of antti-migraine drugs triptans by coacervation method. Asian J Pharm Clin Res 2017; 10:122-8. 